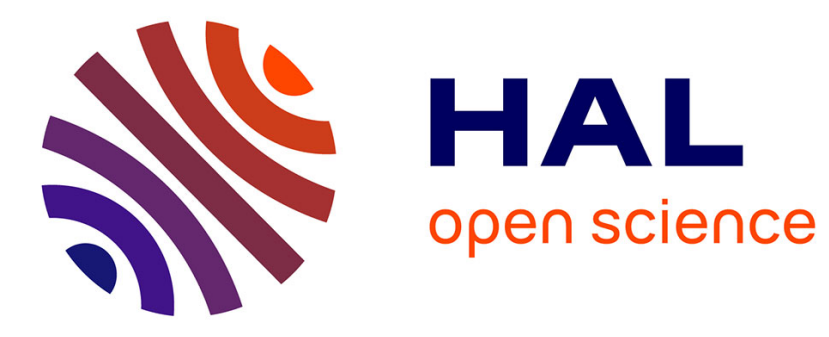

\title{
Modèle " de la poubelle " et dynamique du business model
}

\author{
Vincent Lefebvre, Miruna Radu Lefebvre, Erwan Lamy
}

\section{To cite this version:}

Vincent Lefebvre, Miruna Radu Lefebvre, Erwan Lamy. Modèle " de la poubelle " et dynamique du business model. Entreprendre \& Innover, 2012, 15 (3), pp.77-87. 10.3917/entin.015.0077 . hal00839460

\section{HAL Id: hal-00839460 \\ https://hal-audencia.archives-ouvertes.fr/hal-00839460}

Submitted on 27 Jun 2016

HAL is a multi-disciplinary open access archive for the deposit and dissemination of scientific research documents, whether they are published or not. The documents may come from teaching and research institutions in France or abroad, or from public or private research centers.
L'archive ouverte pluridisciplinaire HAL, est destinée au dépôt et à la diffusion de documents scientifiques de niveau recherche, publiés ou non, émanant des établissements d'enseignement et de recherche français ou étrangers, des laboratoires publics ou privés. 


\section{MODÈLE « DE LA POUBELLE » ET DYNAMIQUE DU BUSINESS MODEL}

Vincent Lefebvre, Miruna Radu Lefebvre, Erwan Lamy

De Boeck Supérieur | «ntreprendre \& Innover »

$2012 / 3 n^{\circ} 15 \mid$ pages 77 à 87

ISSN 2034-7634

ISBN 9782804175788

Article disponible en ligne à l'adresse :

http://www.cairn.info/revue-entreprendre-et-innover-2012-3-page-77.htm

\section{!Pour citer cet article :}

Vincent Lefebvre et al., « Modèle « de la poubelle » et dynamique du business model », Entreprendre \& Innover 2012/3 ( $\left.\mathrm{n}^{\circ} 15\right)$, p. 77-87.

DOI 10.3917/entin.015.0077

Distribution électronique Cairn.info pour De Boeck Supérieur.

(C) De Boeck Supérieur. Tous droits réservés pour tous pays.

La reproduction ou représentation de cet article, notamment par photocopie, n'est autorisée que dans les limites des conditions générales d'utilisation du site ou, le cas échéant, des conditions générales de la licence souscrite par votre établissement. Toute autre reproduction ou représentation, en tout ou partie, sous quelque forme et de quelque manière que ce soit, est interdite sauf accord préalable et écrit de l'éditeur, en dehors des cas prévus par la législation en vigueur en France. Il est précisé que son stockage dans une base de données est également interdit. 


\title{
Modèle " de la poubelle » et dynamique du business model
}

\author{
$>$ Vincent Lefebvre \\ > Miruna Radu Lefebvre \\ > Erwan Lamy
}

\section{Résumé}

Pour élaborer leur modèle d'affaires, les porteurs de projet en situation d'accompagnement sélectionnent certains choix et en écartent d'autres. Sur la base de quels critères ? À quel moment et pour quelles raisons décident-ils ultérieurement d’intégrer certaines options écartées de prime abord ? Pour le comprendre, les auteurs de l'article ont réalisé en 2009 et 2010 une série d'entretiens semi-directifs avec un accompagnateur et trois entrepreneurs dans le cadre de l'Incubateur d'Advancia.

\section{Les points forts}

- Le processus d'élaboration du business model est une co-construction relationnelle et dialogique entre l'entrepreneur et son accompagnateur.

- L'accompagnateur remobilise des exemples de business models déjà rencontrés et disponibles en mémoire à long terme, c'est sa " poubelle à solutions générique ".

- Le créateur d'entreprise mobilise deux systèmes cognitifs interconnectés de " poubelles à solutions " : l'une constituée progressivement en vue de la création et consolidée lors des dialogues avec l'accompagnateur, l'autre ultérieure à l'élaboration du business model de lancement, constituée lors de la phase de développement et composée à la fois des éléments de la poubelle à solutions de la création et de nouveaux éléments rejetés, écartés temporairement mais potentiellement utiles. 
A ssimilé à un " système ${ }^{1}$, une " architecture $\|^{2}$, une " narration $»^{3}$, ou encore un "modèle " cohérent et logique ${ }^{4}$, le business model est un outil clé de la démarche de légitimation ${ }^{5}$, de création de valeur ${ }^{6}$ et de la performance des jeunes entreprises. Lors de son élaboration en situation d'accompagnement, on constate que les entrepreneurs choisissent certaines alternatives relatives aux différentes composantes du business model et à leur articulation, tout en en écartant d'autres. Notre question de départ concerne les processus d'élaboration et d'évolution du business model: sur la base de quels critères l'entrepreneur décide-t-il de retenir certaines alternatives au détriment d'autres ? À quel moment et pour quelles raisons décide-t-il ultérieurement d'intégrer dans le business model de développement certaines options écartées initialement?

Lors de l'élaboration du business model de lancement, l'impossibilité objective de retenir l'ensemble des alternatives possibles conduit inévitablement à des choix non-optimaux d'un point de vue purement rationnel. "Mettre à la poubelle"

1 Zott, C. et Amit, R., Business Model Design and Performance of Entrepreneurial Firms, Organization Science, 2007, 18(2), p. 181-199.

2 Timmers, P., Business models for electronic commerce, Electronic Markets, 1998, 8(2), p. 3-8.

3 Mangematin, V., S. Lemarie, I.P. Boissin, D. Catherine, F. Corolleur, et R. Coronini, Development of SMSs and heterogeneity of trajectories: The case of biotechnology in France, Research Policy, 2003, 32(4), p. 621-638.

4 Chesbrough, H. et Rosenbloom, R.S. The role of the business model in capturing value from innovation: Evidence from Xerox Corporation's technology spin-off companies, Industrial and Corporate Change 2002, 11(3), p. 529-555.

5 Cohen, M.D., March, J.G. et Olsen, I.P. A Garbage Can Model of Organizational Choice, Administrative Science Quarterly, 1972, 17(1), p. 1-25

6 Lounsbury, M. et Glynn, M., Cultural entrepreneurship: Stories, legitimacy, and the acquisition of resources, Strategic Management Journal, 2001, 22, p. 545-564. des options sans les étudier ou les tester systématiquement semble inévitable. Que se passe-t-il avec les alternatives écartées ? Sont-elles rejetées définitivement ou seulement mises de côté et conservées pour une introduction ultérieure, lors de la phase de développement ? Et quel est le rôle de l'accompagnateur dans l'élaboration et l'évolution du business model ? Afin d'explorer les processus d'élaboration et d'évolution du business model en situation d'accompagnement, nous nous inspirons du "modèle de la poubelle " ou Garbage Can Model (GCM) de Cohen, March et Olsen (1972) 7 . Nous avons suivi trois entreprises dans l'Incubateur d'Advancia - CCIP : The Watch observer (2010), Lecointre Paris (2009) et Hal'Shop (2009). Cet article repose sur une série d'entretiens semi-directifs avec les trois entrepreneurs et leur accompagnateur, ainsi que sur l'analyse des business models de lancement et de développement.

Cette recherche permettra aux entrepreneurs de mieux prendre conscience de leurs propres processus de prise de décision les conduisant à choisir certaines solutions dans le cadre du business model de lancement, tout en les aidant, ensuite, à s'appuyer sur certaines solutions écartées initialement afin de mieux rebondir et ajuster leur business model aux opportunités et ressources disponibles. D'autre part, cette recherche permettra aux accompagnateurs de mieux appréhender la connaissance issue des business models déjà rencontrés pour la remobiliser comme ressource en situation d'accompagnement. Après un rappel de la théorie du Garbage Can Model, nous pré-

7 Mahadevan, B., Business models and system architectures of virtual communities: From a sociological phenomenon to peer-to-peer architectures, International Journal of Electronic Commerce, 2000, 6(3), p. 41-53. 
sentons notre cadre d'analyse et les résultats de notre étude exploratoire.

\section{Cadre d'analyse : le modèle de la poubelle}

Partant du constat que la prise de décision repose sur des stimuli ambigus, Cohen, March et Olsen (1972) développent l'idée des " anarchies organisées " pour expliquer la prise de décision dans les organisations. Ce modèle repose sur l'identification de trois propriétés de ces "anarchies ": le système de préférences qui guide les processus de décision est "problématique ", c'est-à-dire qu'il n'est pas toujours cohérent et préexistant aux prises de décision car les préférences se forgent au fil de l'action ; la mécanique décisionnelle est vague et erratique, ses logiques restant souvent opaques aux membres mêmes de l'organisation et reposent essentiellement sur des séries d'essais et d'erreurs, sur divers apprentissages et sur toutes sortes de bricolages; la participation des membres de l'organisation y est fluctuante, l'intensité de leur engagement variant au fil des situations, ce qui brouille les frontières internes et externes de l'organisation et rend difficile sinon impossible de prévoir qui sera engagé dans un processus décisionnel particulier. II s'agit alors de modéliser la manière dont les organisations font des choix en l'absence d'objectifs très clairement définis, et la manière dont les membres de l'organisation peuvent être mobilisés.

Ce modèle est une simulation informatique du processus décisionnel défini comme le résultat du croisement de quatre "flux " indépendants : un flux de problèmes, un flux de solutions, un flux de participants et un flux de choix. Les chercheurs explorent notamment les contours de la notion d'ambiguité ${ }^{8}$, si cruciale pour comprendre le caractère incertain et déstructuré des processus décisionnels dans les organisations.

On retrouve ce modèle aussi bien en sciences de l'organisation, dont il est l'un des piliers, qu'en sociologie et en sciences politiques. Martinet ${ }^{9}$ le compte parmi les trois " paradigmes " de la pensée stratégique, avec la rationalité limitée et la coalition politique. Waguespack ${ }^{10}$ souligne la dimension fondatrice de ce modèle qui serait " un point de départ provocateur dont les idées centrales appellent à être remises en question plutôt qu'à être prises comme un dogme" ".

\section{) Une co-construction entrepreneur- accompagnate ur}

Pour nous, le processus d'élaboration du business model est une co-construction relationnelle et dialogique entre l'entrepreneur et son accompagnateur (voir Figure 1). On envisage ce processus comme un pilotage conjoint d'alternatives reliant opportunités et ressources dans le cadre d'une combinatoire dont le résultat final est co-évalué.

Plus concrètement, en amont de l'accompagnement d'un projet entrepreneurial, l'accompagnateur accumule des connaissances sur les business models existants dans différents secteurs économiques,

8 Cohen, M.D., March, I.G. et Carnegie Commission on Higher Education, Leadership and ambiguity: the American college president, Harvard Business Press, 1986.

9 Martinet, A.-C., James March, un refondateur de la pensée stratégique?, Revue Française de Gestion, 2002, 3-4(139), p. 161-172.

10 Waguespack, D.M., Reconciling garbage cans and rational actors: Explaining organizational decisions about environmental hazard management, Social Science Research, 2006, 35(1), p. 40-59. 
selon son expérience et l'information existante.

De son côté, en amont de l'accompagnement, l'entrepreneur recueille de l'information lui permettant de bâtir son business model initial. Lors de l'accompagnement et en amont du lancement de l'entreprise, l'entrepreneur et l'accompagnateur discutent pour clarifier les composantes du business model (opportunités, ressources, partenaires, proposition de création de valeur), et rendent ainsi explicites les solutions retenues et les options écartées ; c'est lors de cet échange que l'entrepreneur et l'accompagnateur constituent chacun progressivement ce que nous appelons les "poubelles à solutions spécifiques au projet ". Lors de l'accompagnement, l'accompagnateur remobilise des exemples de business models déjà rencontrés par le passé et disponibles en mémoire à long terme, c'est ce que nous appelons " la poubelle à solutions générique » de l'accompagnateur.

Pour résumer, l'accompagnateur mobiliserait dans le cadre de son travail d'accompagnement deux systèmes cognitifs interconnectés de "poubelles à solutions $"$ :

1. une "poubelle à solutions " générique, constituée progressivement dans le temps au fur et à mesure de l'activité d'accompagnement et composée d'éléments rencontrés chez les entrepreneurs suivis - solutions de succès, cas d'erreurs, modalités de tests de solutions, configurations de business models, etc.

2. une "poubelle à solutions" spécifique à chaque projet entrepreneurial, constituée progressivement dans le temps au fur et à mesure des rencontres avec l'entrepreneur et composée d'éléments écartés par le créateur (offre, clients, partenaires, système de distribution, organisation, financement, etc.), et potentiellement remobilisables pour le développement de son entreprise.

Lors de l'accompagnement, l'accompagnateur remobilise des exemples de business models déjà rencontrés par le passé et disponibles en mémoire à longue terme, c'est ce que nous appelons " la poubelle à solutions générique " de l'accompagnateur.

De manière symétrique, l'entrepreneur mobiliserait à son tour deux systèmes cognitifs interconnectés de "poubelles à solutions " spécifiques à son projet :

1. une "poubelle à solutions " de la création, antérieure à l'élaboration du business model de lancement, constituée progressivement en vue de la création et consolidée lors des dialogues avec l'accompagnateur, composée d'éléments rejetés, écartés par l'entrepreneur (offre, clients, partenaires, système de distribution, organisation, financement, etc.).

2. une " poubelle à solutions " du développement, ultérieure à l'élaboration du business model de lancement, constituée progressivement lors de la phase de stabilisation et de développement de l'entreprise et composée à la fois des éléments de la poubelle à solutions de la création et de nouveaux éléments rejetés, écartés temporairement, mais potentiellement utiles (offre, clients, partenaires, système de distribution, organisation, financement, etc.). 


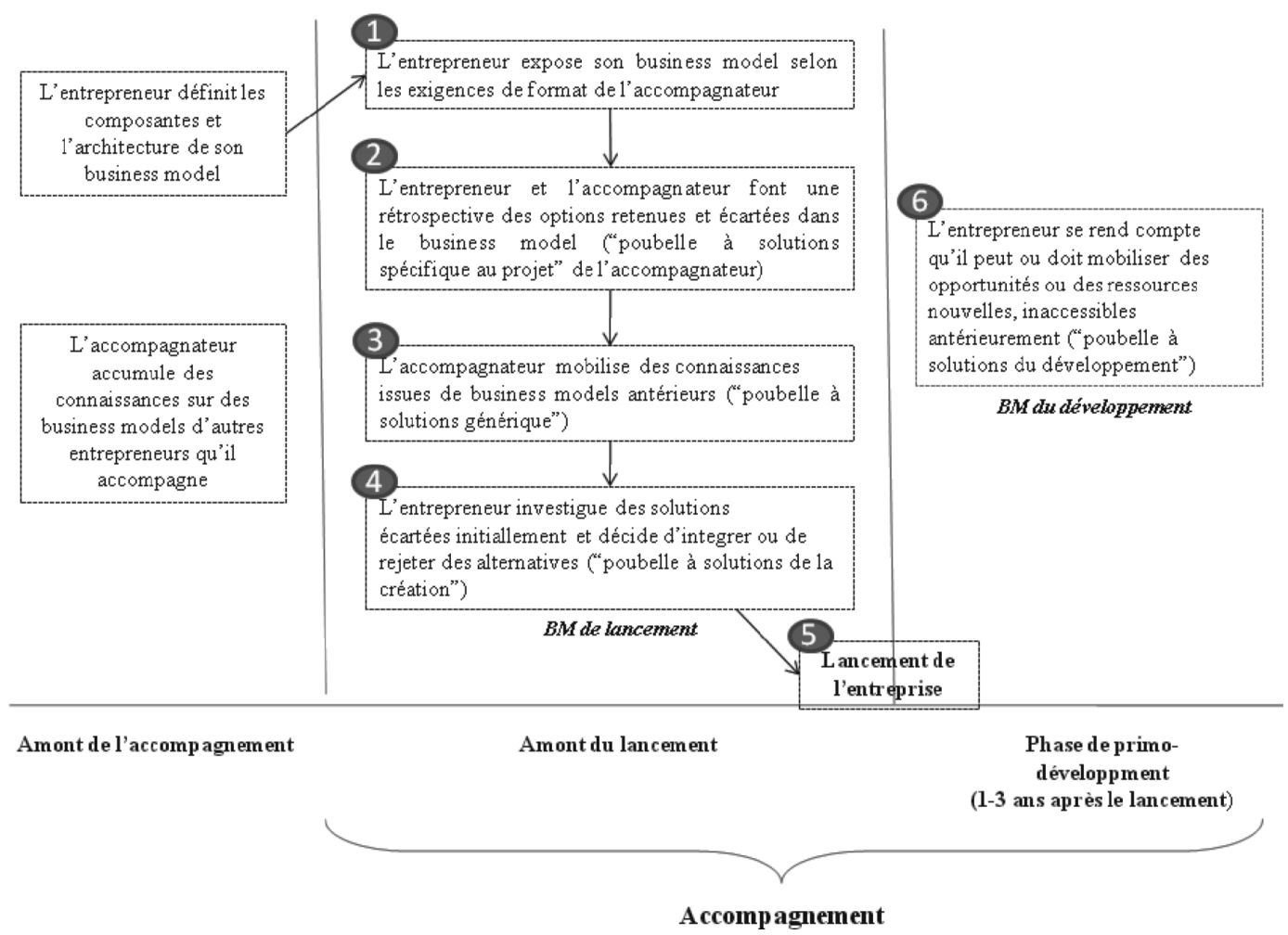

Figure 1 : Élaboration et évolution du business model en situation d’accompagnement

\section{Résultats de notre étude exploratoire}

Cet article repose sur une série d'entretiens semi-directifs avec un accompagnateur et trois entrepreneurs, ainsi que sur l'analyse de trois business models élaborés par les entreprises The Watch observer (2010), Lecointre Paris (2009) et Hal'Shop (2009) dans l'Incubateur d'Advancia, CCIP. Nous reproduisons en encadrés les business models de lancement proposés par les entrepreneurs à l'issue du processus d'accompagnement, ainsi que les business models élaborés lors de la phase de développement. Comment est-on arrivés à ces business models ? Le format utilisé s'apparente à un "Executive summary ", la consigne donnée aux entrepreneurs étant de spécifier les composantes suivantes ainsi que leur articulation : l'opportunité, les ressources, les parties prenantes et la création de valeur.

Nous avons constaté pour les trois cas étudiés que l'accompagnateur remobilise systématiquement des solutions rencontrées chez d'autres entrepreneurs lors du travail d'élaboration du business model. Lors de l'entretien de découverte, l'entrepreneur présente sa proposition de business model, permettant à l'accompagnateur de remonter aux origines de ses choix et de repérer les alternatives sur lesquelles l'entrepreneur a effectué un travail. Les alternatives non-sélectionnées par l'entrepreneur et collectées par l'accompagnateur lors de cet entretien sont classées en deux grandes catégories:

1. Des alternatives intéressantes mais difficiles à mettre en œuvre immédiate- 
ment, à mobiliser éventuellement lors de la phase de développement ;

2. Des alternatives écartées de manière hâtive et qui pourraient être intégrées dans le business model de lancement, car porteuses de valeur ajoutée.

Lors des entretiens ultérieurs, l'accompagnateur tentera de faire intégrer dans le business model les alternatives classées dans le deuxième groupe, tout en essayant de comprendre les raisons qui ont conduit l'entrepreneur à les écarter à l'origine. Comme la situation entrepreneuriale est par nature angoissante ou inconfortable en raison de la prise de risques, l'accompagnateur estime qu'il est important de sécuriser l'entrepreneur et de l'aider à intégrer les alternatives porteuses de valeur ajoutée au moment adapté, tout en respectant ses appréhensions éventuelles.

Par exemple, dans le cas de The Watch Observer des possibilités ont été évaluées en amont du lancement et ont été écartées:

- Faire de la vente de montres en fonction des tests hebdomadaires

- La production de contenu papier

- La vente du contenu à d'autres supports

- La vente de fichier qualifié

- Avoir comme annonceurs des horlogers.

Ces possibilités ont été écartées par l'entrepreneur en amont du lancement pour des raisons de diversification prématurée, de crédibilité ou de notoriété insuffisante. Toutefois, l'accompagnateurabien prévenu l'entrepreneur que ces alternatives étaient à conserver, et éventuellement à mobiliser lors de la phase de développement, et ceci assez rapidement si l'entreprise était appelée ou bien contrainte à les utiliser, pour assurer la croissance ou bien enrayer le risque de faillite par un redéploiement rapide. Lorsque l'entrepreneur est accompagné dans la durée, l'accompagnateur conserve l'historique des échanges. Lors des phases de découverte et de suivi, l'accompagnateur identifie les options envisagées et écartées, ainsi que les étapes de développement et la vision finale de l'entreprise souhaitée par l'entrepreneur. A cet historique qui constitue la " poubelle à solutions spécifique au projet " de l'accompagnateur s'ajoute sa mémoire à long terme (la " poubelle à solutions générique ") permettant de relier des business modèles entre eux et d'accompagner avec un certain recul l'élaboration du business model de lancement.

\section{, Un travail d'exploration et de classement des solutions}

Comment sont concrètement mobilisées les "poubelles à solutions " de l'accompagnateur et de l'entrepreneur ? Dans son travail de généalogie du business model de départ, l'accompagnateur vise à reconstituer la poubelle à solutions de l'entrepreneur et à l'organiser pour une remobilisation ultérieure. Ainsi, l'accompagnateur identifie les alternatives écartées, tente de repérer les raisons de leur rejet, et les reclasse en plusieurs catégories : utiles et inutiles, utiles immédiatement ou utiles ultérieurement.

L'entretien de découverte, et les entretiens suivants (bimensuels) servent de terrain de discussion et de négociation autour de ces classifications de manière à ce que les deux protagonistes partagent les mêmes éléments de la poubelle à solutions spécifique au projet en question. Comment 
l'accompagnateur mène-t-il le travail d'exploration de la poubelle à solutions de l'entrepreneur ? L'identification des alternatives et leur classification est activée de manière intuitive, ceci dépend de l'expertise et de la capacité de l'accompagnateur à relier le projet à d'autres projets similaires soit par type de produit/service, soit par type de transactions envisagées, soit par type de consommateurs visés, soit par d'autres types de critères d'assimilation permettant de faire des transferts de business models entiers ou d'éléments de business models.

L'accompagnateur procède de manière méthodique dans l'examen du business model et fait un travail systématique afin de contourner les blocages de l'entrepreneur et lui montrer que certaines alternatives seraient possibles pour lui, ceci en mobilisant l'ensemble des business models qu'il a pu voir et travailler. Par la suite, lors du travail d'accompagnement, il sera amené à se replonger dans le business model, voire à infléchir les choix de l'entrepreneur lors de situations critiques pour le lancement/la croissance. II plongera ainsi dans sa propre poubelle à solutions générique, ainsi que dans celle spécifique de l'entrepreneur, soit pour en exhumer des éléments intéressants à intégrer pour renforcer le business model de lancement soit pour vérifier que celui-ci n'a pas été arrêté trop hâtivement et que des alternatives potentiellement utiles ont bien été envisagées avant d'être écartées. Ces alternatives seront mobilisables ultérieurement :

- Lors de l'analyse de la faisabilité du projet (l'entrepreneur est-il en capacité de faire ce qu'il propose ?) ;

- Lors de l'analyse de la viabilité du projet (le marché va-t-il répondre favorablement et pourra-t-il vendre à des conditions lui permettant de produire et d'assurer le développement escompté ?) ;

- Lors de la mise en place de la stratégie de développement post création.

En somme, les poubelles à solutions générique et spécifique sont utilisées par l'accompagnateur à chaque fois que la notion de choix est en jeu. Leur usage dépend de la durée de l'accompagnement : si la durée est courte, l'usage de la poubelle à solutions générique est intensif, plus la durée est longue, plus l'usage de la poubelle à solutions spécifique au projet semble important.

\section{? Une remoblisation \\ des solutions écartées}

Notre enquête indique que le rejet de solutions alternatives ne repose pas toujours sur des " tests " préalables. De fait, ily a trop d'options à écarter dans la détermination d'un business model pour examiner l'ensemble des options par une analyse minutieuse. Cependant, certaines alternatives sont testées dans la mesure où l'accompagnateur et/ou l'entrepreneur estiment qu'une investigation est nécessaire afin de renforcer la création de valeur, de stimuler la croissance ou d'enrayer le risque de faillite. Dans le cas des trois entreprises étudiées, on a constaté que des tests ont été effectués avant d'intégrer des alternatives écartées initialement. Dans le cas de Lecointre Paris, certaines options envisagées lors du lancement ont été écartées simplement parce que le temps nécessaire à l'obtention d'une autorisation de longue durée de vente dans l'espace public était trop long et risquait de mobiliser à $100 \%$ l'entrepreneur. 
En somme, lorsque des tests d'alternatives sont effectués, ils reposent rarement sur des démarches très élaborées. L’accompagnateur comme l'entrepreneur s'appuient plutôt sur leur connaissance du marché afin d'identifier aussi vite que possible les informateurs clés permettant de répondre à la question de savoir si l'alternative écartée peut représenter un intérêt immédiat, ultérieur ou nul.

L'accompagnateur et les entrepreneurs ont confirmé que des solutions écartées initialement peuvent être remobilisées et adaptées ultérieurement. L’intégration des solutions écartées initialement peut se produire de deux façons : soit les solutions sont réintégrées jusqu'à modifier de manière significative le business model initial, en amont du lancement, soit elles sont conservées comme mobilisables à condition de rencontrer un environnement favorable pour leur mise en œuvre à des fins de croissance ou de réorientation du business, en aval du lancement. "Les solutions conservées dans la poubelle à solutions de l'entrepreneur comportent en général un potentiel qui ne peut pas être actualisé au démarrage de l'entreprise ", note l'accompagnateur. Lors de leur remobilisation, il est nécessaire de bien identifier quelles sont les conditions de ressources ou d'opportunités qui faisaient barrage initialement. Lorsque l'entrepreneur fait le choix de réintégrer une alternative écartée dans le business model de lancement, c'est parce qu'il a trouvé le moyen de lever une barrière financière, légale ou psychologique au travers de l'accompagnement. Lors de la phase de développement, lorsque l'entrepreneur fait le choix d'intégrer une alternative rejetée initialement, c'est parce que l'immersion dans l'activité a fait émerger de nouvelles possibilités, conduisant à une réévaluation des solutions écartées.

Dans le cas de The Watch Observer, le contact avec les horlogers qui leur prêtent des montres pour faire des tests et l'acquisition d'une notoriété de neutralité dans la notation des montres a conduit l'entrepreneur à accepter de prendre des horlogers comme annonceurs (solution écartée dans le business model de lancement). Également désireux de mener une action promotionnelle au moment de Noël 2010, l'entrepreneur a repris une autre solution écartée, celle relative à d'édition d'un guide, après avoir trouvé un annonceur qui souhaitait financer ce support. Dans le cas de Hal'Shop, l'analyse de la clientèle du magasin a révélé que les consommateurs étaient différents de ceux envisagés à l'origine : il s'agit en réalité de personnes de tradition musulmane, n'habitant pas en centre-ville mais faisant le déplacement pour venir s'approvisionner. La gamme de produits très large comportant des plats de tradition française mais répondant aux exigences Halal n'était pas ou peu consommée. Après ce constat, l'entrepreneur a décidé que les prochains points de ventes seront implantés au plus proche de sa clientèle, dans les quartiers à forte densité de population de tradition musulmane, et avec une gamme de produits restreinte afin d'optimiser le CA et la marge.

Dans le cas de The Watch Observer, l'entrepreneur s'était interdit la vente d'espaces publicitaires à des horlogers afin de conserver son indépendance et de renforcer sa notoriété, fondée sur une image de neutralité. L'adaptation de l'alternative a été contractuelle, "les annonceurs ne bénéficieront d'aucun privilège concernant les tests qui seront réalisés par des 
testeurs indépendants " indique l'entrepreneur (ce n'est plus le site mais ces testeurs qui deviennent indépendants) et d'image : après avoir habitué l'environnement à l'indépendance de l'entreprise, celle-ci est devenue un argument de crédibilité pour les annonceurs horlogers qui n'essayent pas d'influencer les évaluations du site. Dans le cas de Hal'shop, les adaptions ont été multiples et en lien avec toutes les dimensions de la grande distribution : adaptation de la zone de chalandise, de la gamme de produits et de la surface de vente.

Lors de la remobilisation pour enrayer un risque de faillite, le recours à la poubelle à solutions est soudain et discontinu, mais potentiellement bénéfique, car l'actualisation d'une solution écartée se fait alors dans un nouveau contexte, où des informations supplémentaires, voire des compétences nouvelles, sont disponibles. Dans le cas d'une remobilisation de solutions écartées en situation de développement, l'intégration des alternatives peut prendre la forme de paliers lorsque les options conservées dans la poubelle à solutions étaient dès le départ envisagées dans le cadre d'un plan de développement. Dans ce dernier cas, ceci présuppose qu'on avait identifié au préalable les moments clés permettant de les actualiser, soit en raison de l'acquisition de nouvelles ressources, soit en raison du développement d'une nouvelle compétence permettant de répondre à des opportunités.

\section{, Le mot de la fin}

Les implications pratiques de notre recherche concernent à la fois l'entrepreneur et l'accompagnateur. Pour l'entrepreneur, cette recherche pourrait contribuer à une prise de conscience relative aux options écartées lors de l'élaboration du business model de lancement. En effet, il nous semble que certaines options écartées initialement peuvent devenir plus tard des véritables ressources de développement pour l'entreprise. D'où l'importance de consigner ces options lors du travail d'élaboration du business model de création. D'autre part, pour l'accompagnateur, il nous semble utile de souligner le rôle du travail de généalogie du projet lors de la phase de diagnostic, en amont de l'élaboration du business model de lancement. Cette généalogie permet de remonter dans le temps et de comprendre quelles ont été les solutions explorées, retenues et écartées, ainsi que de repérer les raisons pour lesquelles l'entrepreneur a choisi certaines alternatives au détriment d'autres. Ceci permet à l'accompagnateur de lever certains freins et de proposer à l'entrepreneur de réintégrer éventuellement dans le business model de lancement des options écartées trop prématurément. À travers ce travail, l'accompagnateur pourrait ainsi aider l'entrepreneur à constituer sa propre poubelle à solutions spécifique au projet afin de gagner en réactivité et de mieux rebondir lors de la phase de stabilisation et de développement.

Au bout du compte, pour l'entrepreneur comme pour l'accompagnateur, nous pensons nécessaire de ne pas passer trop vite à une formalisation définitive du business model de création, et de prendre le temps nécessaire pour explorer et classer les options relatives aux composantes du business model et à leur articulation. Le business model de lancement devient ainsi un scénario de départ amené à évoluer et dont les options écartées initialement pourraient s'avérer utiles plus tard comme ressources pour la croissance ou la réorientation de l'entreprise. 


\section{Les business models de lancement}

\section{The Watch observer (2010)}

Site internet de contenu éditorial sur les montres s'adressant aux passionnés et aux personnes souhaitant réaliser un achat. Le site fournit des tests réalisés en toute indépendance par rapport aux marques en suivant une grille d'évaluation propre au site et à ses testeurs recrutés parmi des amateurs passionnés d'horlogerie. Le site vend de l'espace publicitaire en s'interdisant les horlogers pour conserver son indépendance. Les visiteurs sont identifiés comme des CSP+ et les annonceurs seront des marques de luxe. Des vendeurs d'horlogerie multimarque sont également visés. L'ensemble des moyens de communication sur le web seront utilisés afin que le nombre de visiteurs uniques par mois augmente rapidement.

\section{Lecointre Paris (2009)}

Adaptation audacieuse à la française du concept de street-vending New-Yorkais de vente de boissons chaudes et froides (café, thé, chocolat et jus de fruits) et de viennoiseries (pain au chocolat, croissant, en plus d'une gamme de sandwich d'un traiteur reconnu). Il s'agit sous une forme raffinée, luxueuse et siglée d'offrir à des points stratégiques (axes majeurs de circulation piétonne et aux pieds des tours de bureaux) de Paris et des villes limitrophes, un service de qualité notamment aux personnes se rendant au travail.

\section{Hal'Shop (2009)}

Hal'Shop est la première chaîne de commerces de proximité 100 \% Halal. Espace moderne, loin des clichés. C'est la solution tant attendue par 5 millions de consommateurs. Ils y découvriront de nouveaux produits alimentaires de qualité, respectant les cultures de chacun. L'espace de vente moderne comportera 300/500 références: produits cuisinés, préparés, surgelés, un espace épicerie et des boissons sans alcool.

Le client cible recherche des produits halal de qualité, pratiques, variés et occidentaux. Parallèlement, d'autres clients sont ciblés : les " touristes alimentaires " qui recherchent un commerce de proximité chaleureux ou des saveurs nouvelles de qualité qui seront proposées à un bon rapport qualité/prix, et qui s'intéressent peu au caractère Halal des produits.

\section{Les business models de développement}

En 2011, à un an et, respectivement, deux ans après le lancement les business models des trois entreprises ont évolué. Nous reproduisons ci-dessous les versions actuelles, avec les éléments nouveaux en italique, et les éléments éliminés par rapport aux business models de lancement indiqués également.

\section{The Watch observer}

Site internet de contenu éditorial sur les montres s'adressant aux passionnés et aux personnes souhaitant réaliser un achat. Le site fournit des tests réalisés par des testeurs indépendants, en toute autonomie par rapport aux marques en suivant une grille d'évaluation propre au site et à ses testeurs recrutés parmi des amateurs passionnés d'horlogerie. Le site étant totalement indépendant dans la rédaction de ses tests de montres vend de l'espace publicitaire. Les visiteurs sont identifiés comme des CSP+ et les annonceurs seront des marques de luxe y compris horlogère. Des vendeurs d'horlogerie multimarque sont également visés. L'ensemble des moyens de communication sur le web seront utilisés afin que le nombre de visiteurs uniques par mois augmente rapidement.

The Watch observer édite avec ses annonceurs un guide annuel de montres au moment de Noël.

\section{Lecointre Paris}

Adaptation audacieuse à la française du concept de street-vending New-Yorkais de vente de boissons chaudes et froides (café, thé, chocolat et jus de fruits) et de viennoiseries (pain au chocolat, croissant, en plus d'une gamme de sandwich d'un traiteur reconnu). 
Il s'agit sous une forme raffinée, luxueuse et siglée d'offrir à des points stratégiques (axes majeurs de circulation piétonne et aux pieds des tours de bureaux) de Paris et des villes limitrophes, un service de qualité notamment aux personnes se rendant au travail.

Cette adaptation du street vending peut également intégrer les entreprises et les espaces foires, salons et congrès et répondre ainsi à des besoins temporaires. Lecointre Paris propose également des prestations de traiteur et de restaurant d'entreprise de petite taille (max. 100 couverts).

\section{Hal'Shop}

Hal'Shop est la première chaîne de commerces de proximité 100 \% halal. C'est la solution attendue par 5 millions de consommateurs. Ils y découvriront de nouveaux produits alimentaires de qualité, respectant les cultures de chacun.

L'espace de vente moderne comportera 100 à 150 références: les produits courants des consommateurs de tradition musulmane. Le client cible recherche des produits halal.

Vincent Lefebvre est professeur en entrepreneuriat à I'ISC Paris, dont il est diplômé, et doctorant au CNAM (Lirsa). Coresponsable de la filière Entrepreneur et de l'incubateur de I'ISC, il travaille dans le champ de l'entrepreneuriat depuis onze ans. Avant de démarrer une carrière académique, il a été notamment Coordinateur régional du dispositif pour la transmission d'entreprises de la CCIP, Responsable du club des entrepreneurs CCIP - Advancia et chef de projets formation au métier d'accompagnateur d'entrepreneurs et de dirigeants. Aujourd'hui, il travaille sur l'utilisation des réseaux formels d'entrepreneurs par les dirigeants membres.

Miruna Radu Lefebvre est professeur associé en entrepreneuriat à Audencia Nantes. Après des études en sciences politiques à I'Université de Bucarest et Sciences Po (IEP - Paris), elle obtient en 2003 le grade de docteur en psychologie sociale de la communication à I'Université Paris 3. Aujourd'hui, elle travaille notamment au développement de recherches qualitatives sur la persuasion et l'en- trepreneuriat. Ses principaux thèmes de recherche sont la représentation sociale de l'entrepreneur et de l'entrepreneuriat, l'impact des modèles de rôles sur l'auto-efficacité et les intentions entrepreneuriales, les stratégies de communication en coaching et mentorat entrepreneurial et leurs effets sur les individus et les entreprises.

Erwan Lamy est épistémologue et sociologue de I'innovation, professeur à Novancia, chercheur associé de I'IDHE Cachan (CNRS, ENS de Cachan). Après une maîtrise de Physique à l'université Paris 6, il obtient en 2005 le grade de docteur en épistémologie, histoire des sciences et des techniques de l'université Paris 7 avec une thèse portant sur les chercheurs entrepreneurs. Aujourd'hui, il travaille notamment au développement d'une épistémologie de l'entrepreneuriat visant à optimiser la qualité des connaissances circulant dans les organisations entrepreneuriales. II accorde une attention toute particulière aux relations entre science et entreprise. 